\title{
A Novel Adaptive Spectrum Defragmentation Algorithm for Elastic Optical Path Networks
}

\author{
Guocheng Wu \\ Engineering Center of Digital Audio and Video \\ Communication University of China \\ Beijing, China \\ cuc_wgc@cuc.edu.cn
}

\author{
Yong Yang \\ Overseas business department \\ Richfit Information Technology Co.,Ltd \\ Beijing, China \\ yangyongrf@sina.cn
}

\begin{abstract}
An efficient spectrum defragmentation algorithm is proposed in elastic optical networks by properly rerouting existing connections after traffic terminated dynamically to improve spectrum efficiency, and shown to be resource efficient.
\end{abstract}

Keywords-optical network; network optimization; Spectrum Defragmentation

\section{INTRODUCTION}

Elastic optical path networks are widely discussed to meet the drastically increasing bandwidth demand. To fully utilize spectrum capacity, connections with different transmission requirements are assigned with number of contiguous frequency slots. A fundamental problem in elastic optical path networks is to route and allocate spectrum resources, which is defined as the routing and spectrum assignment (RSA) problem. Since the connections will be routed depending on the arrival order of demand as well as on the load of the network under dynamic traffic, this will result in a set of connections that will be located on suboptimal paths. As network grows and evolves, necessary network defragmentation operation is needed to reconfigure the network: moving traffic to the optimal available path and freeing up stranded bandwidth throughout the network. Meanwhile, maybe there is no available constitute contiguous spectral bands that satisfy the bandwidth request. The potential enhanced gain brought by elastic mechanism may be limited by the increasingly congested traffic, so spectrum defragmentation would be a powerful auxiliary approach to enhance the ability of elastic optical path networks. A spectrum consolidation scheme is proposed to solve the defragmentation problem by reconfiguring connections to the lower available spectrum in elastic optical path networks [1]. Although the scheme results in better spectrum utilization, still lots of rerouting operations can significant impact network services. To minimize service disruption, a make-before-break rerouting scheme is used to mitigate spectrum fragmentation in distance adaptive SLICE networks [2].

In this work, we propose a dynamic adaptive defragmentation algorithm: Maximum Spectrum-Gain Defragmentation Algorithm (MSGD). Under dynamic traffic, it is possible that a connection is originally established on a suboptimal path, upon tear down of connections and their allocated spectrum resources are released, then that connection can be rerouted to its optimal path and employ less resources. Then free continuous slots on those links are created so that new traffic demands can be accommodated. By evaluating every potential benefit brought by spectrum fragments to existing connections, only the connection with maximum spectrum resource gain will be permitted to utilize the released resources. Simulation results elucidate that the blocking performance is improved significant at the cost of minimum number of interrupted connections. This algorithm reduces the number of operations while retaining the effectiveness of the reconfiguration.

\section{A NOVEL SPECTRUM DEFRAGMENTATION SCHEME}

Spectrum fragments are produced by unpredictable traffic and the corresponding setup and tear down processes of connections. Meanwhile, a connection will hold for a very long period as compared with the connection setup time usually. In this context, we propose defragmentation algorithm that alleviates spectrum fragments in elastic optical path networks.

In this study, we ignore the optical device constraint, the total occupied spectrum resources are defined as the cost of a connection only. We are given a brief mathematical model: $\varphi$ is an arbitrary connection and $\mathrm{H}(\varphi)$ is the hop-count of the connection. $\mathrm{N}(\varphi)$ denotes the number of sub-carriers satisfy the traffic demand of a connection between the source node $S(\varphi)$ to the destination node $\mathrm{D}(\varphi)$. Therefore, the spectrum cost $\mathrm{C}$ of connection $\varphi$ can be defined as: $\mathrm{C}(\varphi)=\mathrm{N}(\varphi) \times \mathrm{H}(\varphi)$. We define $\Delta \mathrm{C}\left(\varphi, \varphi^{\prime}\right)$ is the spectrum resource gain that can be obtained when connection $\varphi$ is replaced by new connection $\varphi^{\prime}$.

$$
\Delta \mathrm{C}\left(\varphi, \varphi^{\prime}\right)=\mathrm{C}(\varphi)-\mathrm{C}\left(\varphi^{\prime}\right)=\left(\mathrm{H}(\varphi)-\mathrm{H}\left(\varphi^{\prime}\right)\right) \mathrm{N}(\varphi)
$$

So connection $\varphi$ is said to be optimal if it is established on the shortest route, the hop-count is minimum and the spectrum cost minimum too. Connections established on their suboptimal path are candidate connections in rerouting process. For example, given a 6-nodes network with the resource utilization (e.g., connection E occupies 4 slots) shown in Figure.1, and assume that the fiber links are bi-directional. The objective is to find potential 
connection which could be rerouted to occupy the released spectrum resources.

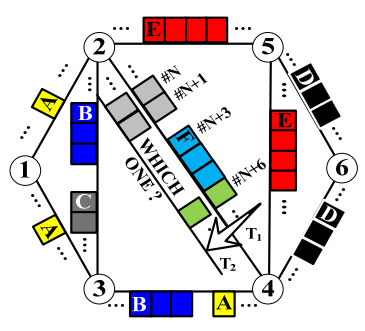

Figure 2. ILLUSTRATIVE

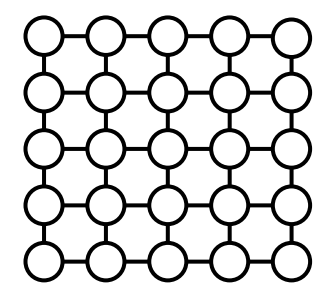

Figure 1. SimUlation TOPOLOGY
TABLE I. POTENTIAL SPECTRUM GAIN

\begin{tabular}{|c|c|}
\hline $\begin{array}{c}\text { Connections } \\
\varphi \rightarrow \varphi^{\prime}\end{array}$ & $\begin{array}{c}\text { Spectrum Gain(Slot) } \\
\Delta C\left(\varphi, \varphi^{\prime}\right)\end{array}$ \\
\hline$\varphi_{\mathrm{A}}: 2-1-3-4 \rightarrow 2-4$ & $(3-1) \times 1=2$ \\
\hline$\varphi_{\mathrm{B}}: 2-3-4 \rightarrow 2-4$ & $(2-1) \times 3=3$ \\
\hline$\varphi_{\mathrm{C}}: 2-3$ & $\begin{array}{c}\text { Already on its } \\
\text { optimal path }\end{array}$ \\
\hline$\varphi_{\mathrm{D}}: 5-6-4$ & No intersection \\
\hline$\varphi_{\mathrm{E}}: 2-5-4 \rightarrow 2-4$ & $(2-1) \times 4=4$ \\
\hline
\end{tabular}

When an existing connection is torn down (e.g. F), in fact, slots from $\# \mathrm{~N}+2$ to $\# \mathrm{~N}+5$ are all free. Existing connections that may give the largest resource gain through rerouting are calculated using formula (1). As shown in Table.1, connection $\mathrm{C}$ could not be chose as the candidate connection since it is already on its optimal path. Because connection D's optimal path (5-4) has no intersection with the torn down path (2-4), connection D isn't the candidate connection either. In our algorithm, the spectrum resource gain indicates the amount of spectrum resources that are returned to the network by the rerouting procedure. Table. 1 implies that connection $\mathrm{E}$ gives the largest resource gain, 4 slots. Thus connection $\mathrm{E}$ is selected to be rerouted to (2-4). Consequently, a large number of low efficient operations are saved, which in turn to decrease senseless reconfiguration and alleviate traffic congestion in elastic optical path networks.

\section{MAXIMUM SPECTRUM-GAIN DEFRAGMENTATION ALGORITHM}

This paper does not consider path set-up or tear down delay, and flooding of updating information is out of scope of this paper. Meanwhile, we assume ROADMs at all nodes are directionless and contentionless with switching ability for contiguous spectrums. Our spectrum defragmentation strategy can be implemented in an ideal central module like Path Computation Element (PCE), where candidate connections are calculated and selected. To focus on the strategy, we also assume that all the network status information including used and available spectrum in every fiber link is collected real-timely by
PCE's Traffic Engineering Database and maintained all the time.

In order to alleviate the impact to the network in rerouting operation, the number of interrupted connections should be minimized. The source node of a connection that is to be torn down does not always initiate the rerouting process, but with probability $\mathrm{R}(0 \leq \mathrm{R} \leq 1)$, namely rerouting rate. The value of $\mathrm{R}$ should be carefully designed. For instance, if $\mathrm{R}$ is too small, few rerouting processes are triggered, that leads to the rerouting process cannot catch up the changing of the network status; if $\mathrm{R}$ is too large, new connections may be forced to use very long path or get blocked. Dynamic rerouting algorithm based on maximum spectrum gain is described at following:

Stage 1: When an existing connection is torn down, the source node sends the rerouting solicit message with routing rate $R$. The request message includes specific available links and corresponding spectrum resources. The value of $R$ is specified manually in this paper, which can also be determined by the condition of the network adaptively.

Stage 2: Find all existing connections that can be rerouted to their optimal paths by using the free up spectrum resources upon receiving a rerouting solicit message. Connections already in their optimal paths, or their optimal paths have no intersection with the released sources would not be considered.

Stage 3: For each candidate connection, network calculates potential spectrum gain using formula (1). Then among the connections, the one with maximum spectrum gain is permitted to be rerouted to its optimal path. In this paper, we only enable at most only one existing connection to be rerouted when a connection is torn down for simplicity.

\section{NUMERICAL EXPERIMENTS AND RESULTS}

To evaluate the performance of the proposed approach, we conducted simulations under the widely used 25 -node polygrid topology (Fig.2). We assumed that each fiber have $4 \mathrm{THz}$ total spectrum and the bandwidth of each slot is $12.5 \mathrm{GHz}$. The required bandwidth for connections was uniformly distributed from 2 to 12 slots. The dynamic connection demands are generated following a Poisson process and the source/destination nodes are assigned randomly. Traffic load (in Erlang) is defined as the average connection arrival rate times connection-holding duration. The holding time of each connection follows a negative exponential distribution. The running time for each evaluation was 500 times the average holding time. We employed Yen's K-Shortest Paths algorithm (KSP) to generate $\mathrm{K}(\mathrm{K}=2)$ candidate routes and First-Fit strategy as spectrum assignment algorithm. Also, we used Connection Blocking Ratio (CBR, the ratio of blocked connections to total connections) to represent the dynamic performance of elastic optical path networks. We compared MSGD algorithm with KSP approach which does not take any further steps after traffic provisioning process in terms of CBR. 


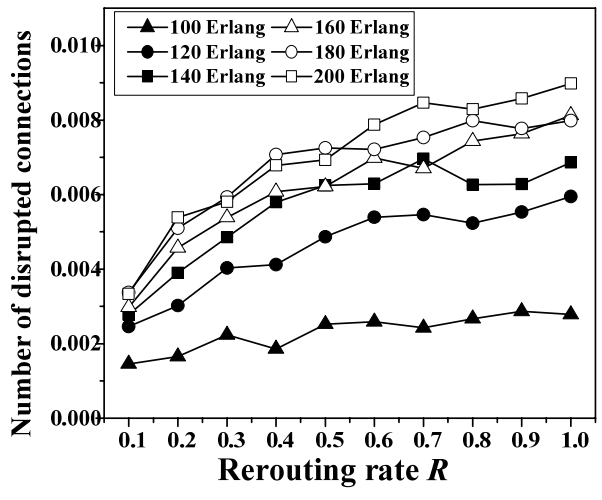

Figure 3. REROUTING RATE VS. INTERRUPTIONS

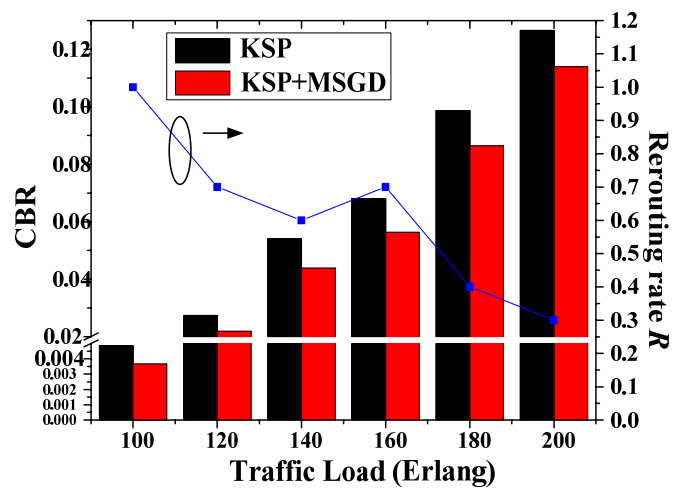

Figure 4. CBR VS. REROUTING RATE

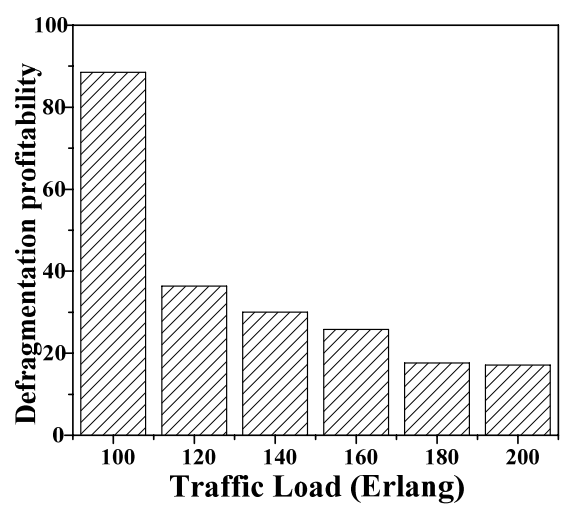

Figure 5. DEFRAGMENTATION PROFITABILITY

Figure.3 illustrates that the number of interrupted connections (normalized to the total number of established connections) in different rerouting rate $\mathrm{R}$. Clearly, the number increases with increasing rerouting rate. As more connections are allocated on their optimal path when the traffic load is light, so the less rerouting opportunities are created. Figure. 4 shows that our dynamic defragmentation algorithm obtains lower blocking probability. It is worth noting that result of fact that the best matched rerouting rate associated with the lowest CBR is various under different traffic load. When the rerouting rate is 1 and the traffic load is 100 Erlangs, CBR can be reduced by more than $24.6 \%$, and its reduction changes from $19.8 \%$ to $9.9 \%$ as the traffic load increases from 120 to 200 Erlangs. At the same time, the rerouting rate fluctuates with the traffic load. The reason is that resource contention takes place more often at higher loads in mesh network, if the rerouting operation is initiated very frequently, existing connections can be routed to their optimal paths. As a result, new connections may be forced to use very long path or get blocked, and then the rerouting rate decreases.

In order to quantitatively describe the benefit of MSGD, we define Profitability of the defragmentation algorithm as the ratio of blocking probability reduction to the ratio of interrupted connections. Fig.5 depicts the defragmentation profitability under different traffic load. It can be observed that MSGD achieves better performance on profitability when the traffic load is light, while the number of the interrupted connections is also less. When traffic load is heavy, little space is provided for the defragmentation, the profitability of the algorithm is weakened.

\section{CONCLUSION}

This paper proposes an efficient spectrum defragmentation algorithm by evaluating potential benefit brought by rerouting of existing connections, while maximizing the amount of resources that can be conserved for future requests. Numerical experiments showed that our algorithm keeps the number of the interrupted connections quite small but it can improve the blocking performance significantly.

\section{REFERENCES}

[1] Jinno M, Takara H, Kozicki B. "Spectrum-efficient and scalable elastic optical path network: architecture, benefits, and enabling technologies," Communications Magazine, 47 (2009), pp.66-73.

[2] Kozicki B, Takara H, Tsukishima Y. "Optical Path Aggregation for 1-Tb/s Transmission in Spectrum-Sliced Elastic Optical Path Network," IEEE Photonics Technol. Lett., 2010, 22(17), pp. 13151317.

[3] Christodoulopoulos K, Tomkos I, Varvarigos E A. "Elastic bandwidth allocation in flexible OFDM-based optical networks, " Journal of Lightwave Technology, 2011, 29(9) , pp. 1354-1366.

[4] Castro A, Velasco L, Ruiz M, et al. "Dynamic routing and spectrum (re)allocation in future flexgrid optical networks," Comput Networks 2012, 56(12), pp. 286-288.

[5] Patel, A.N.; Ji, P.N.; Jue, J.P. "Defragmentation of transparent Flexible optical WDM (FWDM) networks," Optical Fiber Communication Conference (OFC), 2011, pp.1-3, 6-10.

[6] T. Takagi, H. Hasegawa, K. Sato, Y. Sone, A. Hirano and M. Jinno, "Disruption Minimized Spectrum Defragmentation in Elastic Optical Path Network that Adopt Distance Adaptive Modulation", Proc . ECOC. Mo.2.K.3, Sep. 2011.

[7] Ke W, Yawei Y, Geisler J. "Dynamic on-demand lightpath provisioning using spectral defragmentation in flexible bandwidth networks," Proceedings of 37th European Conference and Exhibition on Optical Communication (ECOC), 2011, Geneva, Switzerland, September 2011; Mo.2.K.4. 
[8] J. Wu, "A survey of WDM network reconfiguration: Strategies and triggering methods", Comput. Netw. (2011), pp. 2622-2645.
[9] Gringeri, S.; Basch, B.; Shukla, V. "Flexible architectures for optical transport nodes and networks, "Communications Magazine, IEEE 